\title{
Is it safe to withhold long-term anticoagulation therapy in patients with small pulmonary emboli diagnosed by SPECT scintigraphy?
}

\author{
R. Ghazvinian*, A. Gottsäter and J. Elf
}

\begin{abstract}
Background: The need for anticoagulation therapy (AC) in patients with subsegmental pulmonary embolism (SSPE) diagnosed by computed tomography of the pulmonary arteries (CTPA) has been questioned, as these patients run low risk for recurrent venous thromboembolism (VTE) during 3 months of follow-up. Whether this applies also to patients with small PE diagnosed with pulmonary scintigraphy has not yet been evaluated, however.

Methods: We therefore retrospectively evaluated 54 patients (mean age $62 \pm 19$ years, 36 [67 \%] women) with small PE diagnosed by ventilation/perfusion singe photon emission computed tomography (V/P SPECT) who did not receive conventional long-term AC.

Results: More than half of our patients (36[67 \%]) received less than 48 h of AC, 11 (20\%) patients were treated for 2-14 days, and 7 (13\%) for 15-30 days. The majority (28 [52\%]) of our patients had a non-low simplified pulmonary emboli severity index (S-PESI), and 7 (13\%) had malignancy. D-dimer was negative in 18 (33\%), positive in 10 (19\%), and not analyzed in 28 (52\%) patients. Phlebography of the lower extremities had been performed with negative result in one patient.

During 90 days of follow up no deaths or PE occurred. Seven patients were readmitted to hospital, whereof two (2/54 [4 \%]) were diagnosed with deep venous thrombosis (DVT) necessitating AC therapy.

Conclusion: In conclusion, withholding longterm AC therapy in patients with SSPE diagnosed by V/P SPECT resulted in $4 \%$ risk for recurrence of VTE during 90 days of follow up, and can therefore currently not be recommended.
\end{abstract}

Keywords: Withholding conventional AC therapy, V/P SPECT, Small PE, Subsegmental pulmonary embolism

\section{Background}

The annual incidence rates of deep venous thrombosis (DVT) and pulmonary embolism (PE) are approximately 0.5-1 per 1000 inhabitants [1]. The clinical presentation of $\mathrm{PE}$ extends from asymptomatic patients to shock states, but most patients (95\%) are normotensive and present with breathing difficulties or pleuritic pain [2]. The use of multiple-detector computed tomography pulmonary angiography (CTPA), has led to an increased

\footnotetext{
* Correspondence: Raein.Ghazvinian@skane.se

Lund University, Division of Vascular Medicine, Skåne University Hospital,

Ruth Lundskogs Gata 10, S-205 02 Malmö, Sweden
}

number of diagnosed sub-segmental pulmonary emboli (SSPE), accounting for around 5-15\% of PE cases [3].

Albeit CTPA is considered the primary diagnostic tool for detection of $\mathrm{PE}$ and is available $24 \mathrm{~h}$ a day, 7 days a week, there are alternative methods [4]. Using planar ventilation-perfusion lung scan (V/Q scan), the majority of patients with suspected PE have non-diagnostic examinations [5]. Prospective management studies demonstrated that patients with low or intermediate probability V/Q scan, low pretest probability of PE, and negative compression ultrasonography can be safely managed without anticoagulation [6, 7]. Lung scintigraphy has been further developed, however. Modern ventilation/ 
perfusion single photon emission computed tomography (V/P SPECT) and "holistic" interpretation criteria reduce the risk for non-diagnostic findings, and increases the sensitivity and specificity for diagnosis of PE [8]. With this technique PE can be classified as segmental or subsegmental and graded according to the percentage of the pulmonary vascular bed affected [8].

Some diagnostic challenges persist, however. Even though the use of multi-detector CTPA or V/P SPECT in combination with diagnostic algorithms as for example the Wells score [9] has increased the diagnostic accuracy of PE, the mortality of the disease has remained consistent [10-12]. This might indicate that many of the small SSPE treated after detection with CTPA may be of low clinical significance [4]. In fact, treatment of SSPE in patients with high bleeding risk, and low risk for recurrent venous thromboembolism (VTE) might perhaps even be contraindicated due to increased risk of bleeding and other treatment complications. Whether it is safe to withhold anticoagulant (AC) treatment in patients with SSPE diagnosed by CTPA and negative bilateral ultrasound of the legs is currently evaluated in an ongoing trial [13], but whether this approach is applicable to patients with small PE diagnosed by V/P SPECT has not yet been assessed.

In 898 patients diagnosed with PE by V/P SPECT, we previously reported risk of recurrence and bleeding in 307 patients with small PE undergoing home treatment with AC [14]. We now report a retrospective follow-up of patients with PE diagnosed by V/P SPECT in whom conventional long-term AC therapy for different reasons had been withheld.

\section{Methods}

All consecutive 898 patients with acute PE diagnosed by V/P SPECT between 2007 and 2011 at Lund University Hospital, Sweden were included in a prospective registry [14]. V SPECT had been performed after inhalation of aerosolized $99 \mathrm{~m}$ TC-Technegas to the lung in the supine position with acquisition lasting for $11 \mathrm{~min}$, and $\mathrm{P}$ SPECT with a dual-head gamma camera in the supine position after i.v. administration of $50 \mathrm{MBq} 99 \mathrm{mTc}-$ macroaggregated albumin. V SPECT illuminates ventilated areas and leaves areas with reduced/absent ventilation on the imaging screen, whereas P SPECT illuminates areas where blood flow is normal but leaves areas without perfusion on the imaging screen. PE was diagnosed and quantified by counting segments showing complete or relative mismatch between ventilation and perfusion defects [8].

After diagnosis, patients were clinically assessed according to a pre specified clinical algorithm [14]. Conventional long-term AC treatment defined as therapeutic doses of low molecular heparin or vitamin-K antagonist for at least three months according to current guidelines $[1,12]$ was withheld by the clinician if the V/P SPECT result was interpreted as falsely positive for technical reasons (non diagnostic for PE), if the perfusion defect was thought to represent an old and no longer clinically relevant embolization, or if the embolization was thought to be too clinically irrelevant to merit treatment. Withholding of long-term treatment also required that patients were hemodynamically stable, did not have clinical signs or symptoms of DVT, and that the extent of perfusion defects in the V/P SPECT images was less than $\leq 20 \%$ of the pulmonary vascular bed. Based on these criteria, 54 patients ( $6 \%$, mean age $62 \pm 19$ years, 36 [67\%] women) did not receive conventional long-term $\mathrm{AC}$ therapy. AC treatment of shorter duration was, however, given to many of these patients during the diagnostic work-up as summarized in Table 1 . Baseline data, clinical characteristics including retrospective calculation of sPESI score [15], additional imaging procedures, quantification of perfusion defects on V/P SPECT, d-dimer, and troponin T/ brain natriuretic peptide (NT-BNP) results were collected from patient files. Readmissions, recurrent VTE, and death during 90 days after the final dose of AC therapy were assessed by review of hospital records and imaging databases.

\section{Results \\ Background data}

Twenty (37 \%) patients had a predisposing factor for VTE; active cancer, previous surgery, immobilization, pregnancy, contraceptive pill use, trauma or previous DVT (Table 1). Data on concomitant diseases, sPESI scores, and the number of auxiliary investigations such as D-dimer, compression ultrasound, contrast phlebography and CTPA are also shown in Table 1.

\section{Follow-up}

No deaths occurred during 90 days of follow up after the final dose of AC therapy. Seven patients (13\%) were readmitted to hospital, in 5 cases $(9 \%)$ for suspected VTE. One patient (2\%) underwent CTPA, without signs of PE. Four patients $(7 \%)$ underwent phlebography or ultrasound of the lower extremities, whereof two (2[4\%]) were diagnosed with DVT necessitating long-term AC therapy (Fig. 1).

One 71 year old man who had received AC for $24 \mathrm{~h}$ was readmitted 38 days after the final AC dose because of swelling of the left leg, and ultrasound showed DVT extending up to the external iliac vein provoked by plaster cast immobilization due to a tibial fracture. One 92 years old woman who had received AC for 20 days was readmitted 52 days after the final dose of $\mathrm{AC}$ therapy due to swelling of the right leg. Ultrasound showed DVT extending up to the common femoral vein. 
Table 1 Baseline characteristics and duration of anticoagulant (AC) therapy in 54 patients with small pulmonary embolism diagnosed by ventilation/perfusion single photon emission computed tomography (V/P SPECT) who did not receive conventional long-term AC. N(\%)

Predisposing factors for thrombosis $\mathrm{n}(\%)$

\begin{tabular}{|c|c|}
\hline Malignancy & $7(13)$ \\
\hline Oral contraception & $1(2)$ \\
\hline Surgery or immobilization & 5 (9) (one with malignancy) \\
\hline Trauma & $1(2)$ \\
\hline $\begin{array}{l}\text { Previous venous } \\
\text { thromboembolism }\end{array}$ & $4(7)$ \\
\hline Pregnancy & $2(4)$ \\
\hline \multicolumn{2}{|l|}{ Concomitant diseases n(\%) } \\
\hline Congestive heart failure & $18(33)$ \\
\hline $\begin{array}{l}\text { Chronic obstructive pulmonary } \\
\text { disease or asthma }\end{array}$ & $9(17)$ \\
\hline \multicolumn{2}{|l|}{ Investigations n(\%) } \\
\hline D-dimer & $\begin{array}{l}28(52) \text {, negative in total } 18 \\
\text { (33) }\end{array}$ \\
\hline NT-proBNP & 9 (17), negative in total 8 (15) \\
\hline Troponin T & $\begin{array}{l}28(52), \text { negative in total } 24 \\
\text { (44) }\end{array}$ \\
\hline $\begin{array}{l}\text { Venous ultrasound or } \\
\text { phlebography }\end{array}$ & $1(2)$, negative in total $1(2)$ \\
\hline CTPA & 17 (31), positive in 6 (11) \\
\hline \multicolumn{2}{|l|}{ Risk stratification n(\%) } \\
\hline sPESI score 0 & $12(22)$ \\
\hline sPESI score 1 & $28(52)$ \\
\hline sPESI score 2 & $11(20)$ \\
\hline sPESI score 3 & $2(4)$ \\
\hline sPESI score 4 & $1(2)$ \\
\hline \multicolumn{2}{|c|}{ Duration of anticoagulant therapy n(\%) } \\
\hline$<48$ hours & $34(63)$ \\
\hline 2-14 days & $11(20)$ \\
\hline 15-30 days & $7(13)$ \\
\hline 31-90 days & $2(4)$ \\
\hline
\end{tabular}

\section{Discussion}

To our knowledge, this is the first retrospective study in which withholding of conventional long-term AC therapy has been clinically evaluated in patients with a diagnosis of small PE made by V/P SPECT. We report two cases of DVT during a follow up period of three months (4\%), but no PE or death. These figures are comparable higher than what has been reported when AC therapy has been withheld in SSPE patients diagnosed by CTPA. Goy et al retrospectively study reported no recurrences (0 \%) among 30 patients but that treatment provoked major bleeding in 2/ 43 patients treated with AC [3]. Moreover, Carrier and co-

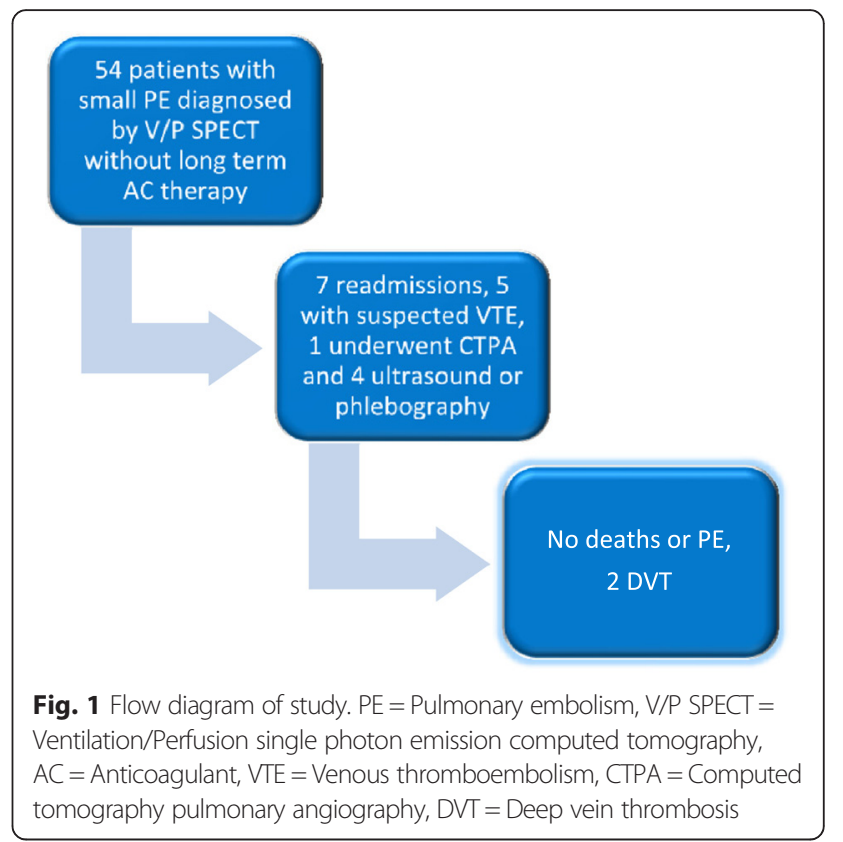

workers summarized no recurrences in 60 patients with SSPE diagnosed with CTPA in combination with a negative compression ultrasonography [4], when reviewing data from four different reports [10,16-18]. These low numbers must be compared with the results of den Exter et al who reported that 4/116 (3.5\%) patients with SSPE treated with AC had a recurrent VTE during 3 months follow up challenging the assumption that SSPE patients have a low risk profile and better clinical outcome compared to patients with more proximal PE [19].

In a cross-sectional survey on clinician's opinions on SSPE [20], it was shown that physicians are comfortable with withholding of therapy if the 3 month risk for recurrent VTE is $<2 \%$. This means that our algorithm with a cutoff of $<20 \%$ extension of perfusion defects in the V/P SPECT images leads to a rate of VTE recurrence that would not be considered acceptable for the majority of clinicians. Whether the use of a lower limit of PE extension on V/P SPECT such as for example $<5 \%$ or $<10 \%$ would have resulted in a lower risk for recurrent DVT remains to be systematically investigated. The extension of the perfusion defects on V/P SPECT in our two patients later diagnosed with VTE was $<10 \%$, and use of this cut-off would therefore not have resulted in any VTE diagnoses during follow-up in our material.

Whether a normal result on bilateral lower extremity ultrasonography should be requested before AC therapy is withheld in patients with confirmed small PE on V/P SPECT also needs to be further evaluated. Whereas this strategy has been used after SSPE diagnosis with CTPA [3] and is currently under further scientific evaluation [13], none of our two patients later diagnosed with DVT had undergone ultrasound or phlebography when the 
decision to abstain from long-term AC therapy was made.

Whether results of the s-PESI score [17] can be used to predict which patient with small PE that will develop VTE during follow-up is still unclear. Retrospectively calculated s-PESI scores differed in the two patients with VTE during follow-up in our study; zero in the male patient and three in the female patient. Our male patient had an elevated D-dimer which was thought to be caused by concomitant disease, whereas D-dimer was not assessed in the female patient. The combination of Wells score [9] and D-dimer in the diagnostic work-up of $\mathrm{PE}[1,12]$ is only applicable in outpatients without too many comorbidities.

The results of our study underlines the importance of the ongoing prospective study [13] performed to evaluate the safety of withholding $\mathrm{AC}$ therapy in patients with SSPE. It is important to note, however, that the results of this trial will only be applicable in patients in whom PE is diagnosed by CTPA and in whom serial ultrasound of the lower extremities is negative. Our results indicate that the future study results cannot be extrapolated to patients with small PE diagnosed by V/P SPECT, at least not without routinely performing bilateral compression ultrasonography of the lower extremities.

The discrepancies in results between our and previous $[3,4,10,16-19]$ might also be caused by differences in the composition of the study populations. When relating our results to previously published data $[10,15-17,21]$, our patients were of comparable age (62 years vs 65 and 56 years in the studies of Goy et al. [3] and den Exter et al [19]). The prevalence of malignancy was lower in our study (13\% vs 28 and $18 \%$ ) respectively, however. Furthermore, one third of our patients had congestive heart failure (CHF) and $17 \%$ had chronic obstructive pulmonary disease (COPD), figures higher than the $9 \%$ for both CHF and COPD reported by den Exter et al. [19].

Among patients with malignancies and $\mathrm{PE}$, the risk for recurrent VTE is increased among those with symptomatic PE, compared to those with incidentally detected asymptomatic disease [20]. In our study, however, no VTE was detected during follow-up of the 7 patients with known malignancies.

The duration of AC treatment given during diagnostic work-up and initial hospital care is another important issue. In the majority $(63 \%)$ of our patients who received less than $48 \mathrm{~h}$ of AC therapy, one case of VTE occurred, whereas the other case of VTE was diagnosed in the group (13\%) of patients receiving 15-30 days of AC therapy. The risk of recurrence during the first three months is approximately $2 \%$ [22], and the concept of generally prescribing one month of $\mathrm{AC}$ to patients diagnosed with small $\mathrm{PE}$ with V/P SPECT, thereby limiting the risk of bleeding, also needs to be further investigated.
The aim of our retrospective study was not to compare V/P SPECT with CTPA in diagnosis of small PE, but PE diagnosis made on V/P SPECT nevertheless had been reassessed with CTPA in $31 \%$ of patients, and was confirmed in a third of these. This reflects that the clinical decision to withhold long term AC therapy was made in three different situations after a positive $\mathrm{V} / \mathrm{P}$ SPECT; when the result was believed to be non diagnostic, when the perfusion defect was thought to represent a no longer clinically relevant embolization, and when the embolization was thought to be clinically irrelevant. The reason to perform CTPA is most evident in the first of these three scenarios, and in 11 of our patients the PE diagnosis was dropped after a negative CTPA. In the 6 patients in whom long-term AC therapy was withheld in spite of positive results on both V/P SPECT and CTPA, therapy was withheld by the clinician for the third of the above reasons.

A recent paper comparing diagnostic performance between CTPA and V/P SPECT reported that these modalities were equivalent with respect to receiver operating curves (ROC) analysis, with areas under curve (AUC) of 0.99 (95 \% CI 0.96-1.00) and 0.98 (95 \% CI 0.94-1.00) for V/P SPECT and CTPA, respectively [23].

The main study limitation is of course that we conducted a retrospective clinical follow-up, and not a randomized prospective study. Furthermore, as the decision to withhold conventional long-term AC therapy was made on different ground, our patient material is heterogeneous. Nevertheless, we think that it represents practice in an often occurring difficult clinical situation.

\section{Conclusions}

Withholding of conventional long term AC therapy in patients diagnosed with small PE with V/P SPECT was associated with a $4 \%$ risk of VTE diagnosis during 3 months of follow-up. This would not be considered acceptable for the majority of clinicians, and the concept can at the present stage therefore not be recommended.

\section{Abbreviations \\ $\mathrm{AC}$, anticoagulation; $\mathrm{AUC}$, area under curve; $\mathrm{CHF}$, chronic heart failure; $\mathrm{COPD}$, chronic obstructive pulmonary disease; CTPA, computed tomography pulmonary arteries; DVT, deep vein thrombosis; $\mathrm{PE}$, pulmonary embolism; ROC, reciever operating curves; S-PESI, simplified pulmonary emboli severity index; SSPE, subsegmental pulmonary embolism; V/P SPECT, ventilation/perfusion single photon emission computed tomography; V/Q scan, ventilation-perfusion lungscintigraphy; VTE, venous thromboembolism.}

\section{Acknowledgement}

None.

Funding

No funding was obtained for this article.

Availability of data and supporting materials

The unidentified dataset supporting the conclusions of this article is available upon request by contacting the corresponding author. 


\section{Authors' contributions}

RG and JE contributed to the study concept and design, and acquisition of the data. RG, AG, and JE all contributed to data analysis and interpretation, and drafting and critical revision of the manuscript. Statistical analysis was performed by RG.

\section{Competing interests}

The authors state that they have no conflicts of interests.

\section{Consent for publication}

Not applicable.

\section{Ethics approval and consent to participate}

The local Ethics committee of Lund University approved the study 26 Aug 2011, Dnr 2011/388

Received: 5 February 2016 Accepted: 25 May 2016

Published online: 31 May 2016

\section{References}

1. Torbicki A, Perrier A, Konstantinides S, Agnelli G, Galiè N, Pruszczyk P, Bengel F, Brady AJ, Ferreira D, Janssens U, Klepetko W, Mayer E, Remy-Jardin M, Bassand JP. Guidelines on the diagnosis and management of acute pulmonary embolism: the Task Force for the Diagnosis and Management of Acute Pulmonary Embolism of the European Society of Cardiology (ESC). Eur Heart J. 2008;29(18):2276-315.

2. Kasper W, Konstantinides S, Geibel A, Olschewski M, Heinrich F, Grosser KD, Rauber K, Iversen S, Redecker M, Kienast J. Management strategies and determinants of outcome in acute major pulmonary embolism: results of a multicenter registry. J Am Coll Cardiol. 1997;30(5):1165-71.

3. Goy J, Lee J, Levine O, Charudhry S, Crowther M. Sub-segmental pulmonary embolism in three academic teaching hospitals: a review of management and outcomes. J Thromb Haemost. 2015;13:214-8.

4. Carrier M, Righini M, Le Gal G. Symptomatic subsegmental pulmonary embolism: what is the next step? J Thromb Haemost. 2012;10:1486-90.

5. Stein PD, Henry JW. Prevalence of acute pulmonary embolism in central and subsegmental pulmonary arteries and relation to probability interpretation of ventilation/perfusion lung scans. Chest. 1997;111:1246-8.

6. Perrier A, Bounameaux H, Morabia A, de Moerloose P, Slosman D, Didier D, P-f U, Junod A. Diagnosis of pulmonary embolism by a decinsion analysisbased strategy including clinical probability, D-dimer levels, and ultrasonography: a management study. Arch Intern Med. 1996;156:531-6.

7. Salaun PY, Couturaud F, Le Duc-Pennec A, Lacut K, Le Roux PY, Guillo P, Pennec PY, Cornily JC, Leroyer C, Gal G. Noninvasive diagnosis of pulmonary embolism. Chest. 2011;139:1294-8.

8. Bajc M, Neilly JB, Miniati M, Scheumichen C, Meignan M, Jonson B. EANM guidelines for ventilation/perfusion scintigraphy: Part 1. Pulmonary imaging with ventilation/perfusion single photon emission tomography. Eur J Nucl Med Mol Imaging. 2009;36:1356-70.

9. Wells PS, Anderson DR, Rodger M, Stiell I, Dreyer JF, Barnes D, Forgie M, Kovacs G, Ward J, Kovacs MJ. Excluding pulmonary embolism at the bedside without diagnostic imaging: management of patients with suspected pulmonary embolism presenting to the emergency department using a simple clinical model and d-dimer. Ann Intern Med. 2001;135:98107.

10. Pena E, Kimton M, Dennie C, Peterson R, Le Gal G, Carrier M. Difference in interpretation of computed tomography pulmonary angiography diagnosis of subsegmental thrombosis in patients with suspected pulmonary embolism. J Thromb Haemost. 2012;10:496-8.

11. Wiener R, Schwartz LM, Woloshin S. Time trends in pulmonary embolism in the United States: evidence of overdiagnosis. Arch Intern Med. 2011;171:831-7.

12. Kearon C, Akl EA, Comerota AJ, Prandoni P, Bounameaux H, Goldhaber SZ, Nelson ME, Wells PS, Gould MK, Dentali F, Crowther M, Kahn SR. Antithrombotic therapy for VTE disease: antithrombotic therapy and prevention of thrombosis, 9thed: American college of chest physicians evidence-based clinical practice guidelines. Chest. 2012;141:419S-94.

13. A study to evaluate the safety of withholding anticoagulation in patients with subsegmental PE who have a negative serial bilateral lower extremity ultrasound (SSPE). https://clinicaltrials.gov/ct2/show/NCT01455818. Accessed at Aug 13, 2014
14. Elf J, Jögi J, Bajc M. Home treatment of patients with small to medium sized acute pulmonary embolism. J Thromb Thrombolysis. 2015;39:166-72.

15. Masotti L, Panigada G, Landini G, Pieralli F, Corradi F, Lenti S, Migliacci R, Arrigucci S, Frullini A, Bertieri MC, Tatini S, Fortini A, Cascinelli I, Mumoli N, Giuntoli S, De Palma A, Crescenzo V, Piacentini M, Tintori G, Dainelli A, Levantino G, Fabiani P, Risaliti F, Mastriforti R, Voglino M, Carli V, Meini S. Simplified PESI score and sex difference in prognosis of acute pulmonary embolism: a brief report from a real life study. J Thromb Thrombolysis. 2015; 9:1-7.

16. Donato AA, Scheirer JJ, Atwell MS, Gramp J, Duszak Jr R. Clinical outcomes in patients with suspected acute pulmonary embolism and negative helical computed tomographic results in whom anticoagulant was withheld. Arch Intern Med. 2003;163(17):2033-8.

17. Eyer BA, Goodman LR, Washington L. Clinicians' response to radiologists' reports of isolated subsegmental pulmonary embolism or inconclusive interpretation of pulmonary embolism using MDCT. AJR Am J Roentgenol. 2005;184(2):623-8.

18. Carrier M, Kimpton M, Le Gal G, Kahn SR, Kovacs MJ, Wells PS, Anderson DR, Rodger MA. The management of a sub-segmental pulmonary embolism: a cross-sectional survey of Canadian thrombosis physicians. J Thromb Haemost. 2011;9:1412-5.

19. den Exter PL, Van Es J, Klok AF, Kroft LJ, Kruip MJHA, Kamphuisen PW, Büller, Huisman MV. Risk profile and clinical outcome of symptomatic subsegmental acute pulmonary embolism. Blood. 2013;122(7):1144-9.

20. den Exter PL, Hooijer J, Dekkers OM, Huisman MV. Risk of recurrent venous thromboembolism and mortality in patients with cancer incidentally diagnosed with pulmonary embolism: a comparison with symptomatic patients. J Clin Oncol. 2011;29:2405-9.

21. Le Gal G, Righini M, Parent F, van Strijen M, Couturaud F. Diagnosis and management of subsegmental pulmonary embolism. J Thromb Haemost. 2006:4:724-31.

22. Prins $M H$, Lensing AW, Bauersachs $R$, van Bellen $B$, Bounameaux $H$, Brighton TA, Cohen AT, Davidson BL, Decousus H, Raskob GE, Berkowitz SD, Wells PS. Oral rivaroxaban versus standard therapy for the treatment of symptomatic venous thromboembolism: a pooled analysis of the EINSTEIN-DVT and PE randomized studies. Thromb J. 2013;11:21.

23. Phillips JJ, Straiton J, Staff RT. Planar and SPECT ventilation/perfusion imaging and computed tomography for the diagnosis of pulmonary embolism: a systematic review and meta-analysis of the literature, and cost and dose comparison. Eur J Radiol. 2015;84(7):1392-400.

\section{Submit your next manuscript to BioMed Central and we will help you at every step:}

- We accept pre-submission inquiries

- Our selector tool helps you to find the most relevant journal

- We provide round the clock customer support

- Convenient online submission

- Thorough peer review

- Inclusion in PubMed and all major indexing services

- Maximum visibility for your research

Submit your manuscript at www.biomedcentral.com/submit
) BioMed Central 